\title{
Genetics of inherited retinal disease
}

\author{
Peter J Francis
}

J R Soc Med 2006;99:189-191

SECTION OF OPHTHALMOLOGY

\section{INTRODUCTION}

The introduction of ophthalmoscopy towards the end of the 19th century afforded physicians for the first time detailed views of the fundus of the eye. Over the subsequent 150 years, a huge number of articles have been dedicated to the description of the various patterns of hereditary retinal diseases, also known as retinal dystrophies. Advances over the last two decades in gene identification and cell biology techniques have led to a step change in our understanding providing real insights into the genetic defects and molecular pathophysiological mechanisms that underlie these conditions. Only now, at the beginning of the 21st century, are there realistic prospects that these blinding conditions may be cured.

The retina is a wonderful tool for the study of genetic disease for several reasons. Not only can a wide-variety of phenotypes be directly visualized and documented photographically but their effects on the organ can be quantified by psychophysical measures (acuity, field, colour contrast) and retinal electrophysiology (by which it is now possible to dissect the function of each retinal cell type individually). Since retinal dystrophies are slowly progressive and may not become visually handicapping until adult life, families with these conditions often become large enough for genetic linkage studies. Finally, the ease with which the retina can be visualized has led to the discovery of a large number of animal models which in turn have led to a better understanding of the genetic and biochemical mechanisms resulting in photoreceptor death. ${ }^{1}$

\section{METHODS}

The articles reviewed in the article were obtained by using key word and the search engine PubMed [www.pubmed.com].

\section{INHERITED RETINAL DYSTROPHIES}

Retinitis pigmentosa is the most common retinal dystrophy affecting 1 in 3000 individuals. Family studies of its Xlinked form were among the first to identify a mutation responsible for a human disease. Retinitis pigmentosa is now known to be remarkably genetically heterogeneous with over 150 mapped chromosomal loci and one hundred

Eye Department, St Thomas' Hospital, Lambeth Palace Road, London SE1 7EH, UK

E-mail: peter.francis@gstt.sthames.nhs.uk genes responsible for the phenotype [http://www.sph. uth.tmc.edu/RetNet/]. Thus, rather than being considered a single phenotypic entity unified by the presence nightblindness, progressive visual field loss, eventual acuity loss and bone-spicule retinal pigmentation, the retinitis pigmentosa phenotype is now considered a final common clinical pathway that arises from a number of insults that lead to rod photoreceptor degeneration. Most retinitis pigmentosa mutations affect rods selectively. The function of the genes involved is highly varied, from those that encode proteins for phototransduction to structural and transmembrane proteins and even transcription factors. By pathways which at the moment remain obscure, each leads to rod photoreceptor cell death by apoptosis, a mechanism of programmed cell death. Interestingly, cones are seldom directly affected by the mutations that cause retinitis pigmentosa but appear to degenerate secondarily to rods, accounting for the loss of central vision and complete blindness sometimes seen at the end-stage of the disease. ${ }^{2}$

Leber congenital amaurosis is the severest of all retinal dystrophies. Affected individuals usually present in the first year of life with profound blindness, roving nystagmus, variable retinal pathology and occasionally other systemic pathology. In contrast to original thinking based on an apparently characteristic and unifying phenotype, Leber congenital amaurosis is now known to be caused by at least six genes. To add further complexity, different mutations in several of these genes have been observed to cause retinitis pigmentosa and other retinal dystrophies. It is suggested that the Leber congenital amaurosis phenotype must therefore result from mutations which have the severest consequence. ${ }^{3}$ One of the genes, RPE65, has become of particular significance following the finding of a spontaneously occurring mutation in the equivalent gene in the Briard dog. Trials of gene replacement therapy have proved successful in restoring sight to such affected dogs and in the next couple of years the first human trials of gene therapy by replacing human photoreceptor RPE65 are timetabled. ${ }^{4}$

\section{DYSTROPHIES OF THE MACULA}

By contrast with retinitis pigmentosa, in which degeneration of the rods initially affects the function of the peripheral retina by virtue of their anatomical location, a large number of inherited retinal dystrophies primarily affect the macula, the part of the retina specialized for 

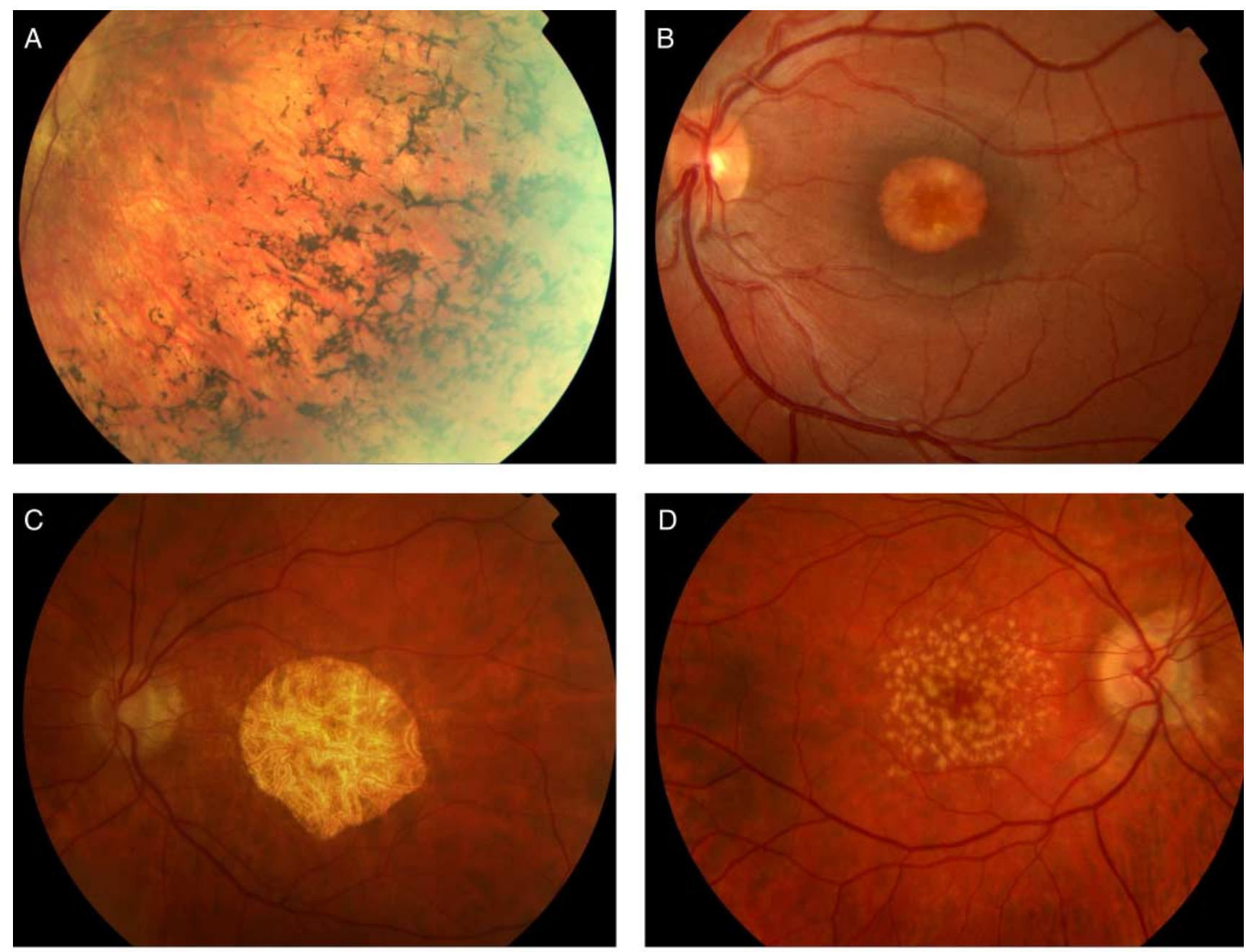

Figure 1 (a) Typical bone-spicule intra-retinal pigmentation in a patient with retinitis pigmentosa; (b) vitelliform macular dystrophy; (c) central areolar choroidal sclerosis; (d) dry age-related macular degeneration (AMD) showing extensive macular drusen [in colour online]

central vision and advanced visual function. These 'macular dystrophies' are characterized by gradual loss of acuity, colour vision and contrast sensitivity with onset usually by the second decade of life. The clinical appearances are markedly heterogeneous and have attracted a wide variety of highly descriptive and often overlapping names including pattern, vitelliform and North Carolina macular dystrophies, fundus flavimaculatus, and central areolar choroidal dystrophy (Figure 1). Perhaps a classification based upon molecular pathology would be more satisfactory however research into the molecular genetic basis of these relatively rare disorders is at a fairly rudimentary stage but with potentially great significance. ${ }^{5}$

\section{AGE-RELATED DEGENERATION OF THE MACULA}

Age-related macular degeneration is the leading cause of blindness in the Western World. Its incidence is expected to double in the next three decades. Currently, treatment and laser therapy, but most patients nevertheless experience progressive visual loss. The disease's hallmark is the subretinal accumulation of lipid and protein-containing deposits known as drusen at the macula. Subsequently, vision is lost because the macula becomes degenerate, either atrophic (dry) or scarred following neovascularization invading from the underlying choroid (wet age-related macular degeneration). ${ }^{6}$

Studies indicate age-related macular degeneration has a strong genetic component. Epidemiological, twin and family studies indicate that in most cases age-related macular degeneration is transmitted as a complex trait, a condition in which the interplay of multiple susceptibility genes and environmental factors determines an individual's risk of developing the disease. ${ }^{7}$ Genes implicated in monogenic macular dystrophies are thus good candidate susceptibility genes for age-related macular degeneration; although to date, with the possible exception of ABCA4, none of these genes have been shown to confer increased risk of developing the condition. 


\section{FUTURE DEVELOPMENTS}

The next tentative steps for retinal genetics lie in the development of treatments and cures for these blinding disorders. As has been mentioned above, they are good targets for gene therapy. The eye and retina are easily accessible but isolated from the rest of the body and photoreceptors appear readily transduceable with genetic material using a variety of virus-based vectors. The X-linked and recessive varieties of disease represent the best candidates for treatment producing the most severe and early onset phenotypes but demanding only gene replacement of the missing gene to be potentially curative. The treatment of dominant retinal dystrophies will be more challenging as the function of the defective protein produced by the mutation needs to be knocked out. More speculative strategies currently under investigation to treat retinal dystrophies include the use of growth factors to slow down or even stop the process of photoreceptor degeneration $^{8}$, cell replacement techniques using transplantation or stem cell technology ${ }^{9}$ and even the development of silicon-based technology to create an artificial retina. ${ }^{10}$ The hope must be that these studies will not only benefit those currently blinded by retinal dystrophies but that the techniques and knowledge will be applicable to other aspects of, and more common, human disease.

\section{CONCLUSION}

Retinitis pigmentosa is the common inherited disorder of the retina and is now known to be caused by a large number of genes with differing functions. Age-related macular degeneration which has a genetic basis is the most common cause of blindness in the Western World. The genes that cause inherited dystrophies of the macula have been considered good candidate genes for age-related macular degeneration though with one exception none appear to confer disease susceptibility. In the next few years, gene therapy is likely to be introduced for some of the most severe retinal dystrophies.

Competing interests None declared.

\section{REFERENCES}

1 Pacione LR, Szego MJ, Ikeda S, et al. Progress toward understanding the genetic and biochemical mechanisms of inherited photoreceptor degenerations. Ann Rev Neurosci 2003;26:657-700

2 Delyfer MN, Leveillard T, Mohand-Said S, et al. Inherited retinal degenerations: therapeutic prospects. Biol Cell 2004;96:261-9

3 Francis PJ, Weleber RG, Trzupek KM. Leber Congenital Amaurosis. In: GeneReviews at GeneTests: Medical Genetics Information Resource. Seattle: University of Washington, 1997-2004 h [www.genetests.org]

4 Koenekoop RK. An overview of leber congenital amaurosis: a model to understand human retinal development. Surv Ophthalmol 2004;49:379-98

5 Michaelides M, Hunt DM, Moore AT. The genetics of inherited macular dystrophies. J Med Genet 2003;40:641-60

6 Klein ML, Francis PJ. Genetics of age-related macular degeneration. Ophthalmol Clin North Am 2003;16:567-74

7 Hammond CJ, Webster AR, Snieder H, et al. Genetic influence on early age-related maculopathy: a twin study. Ophthalmology 2002;109:730-6

8 Chaum, E. Retinal neuroprotection by growth factors: a mechanistic perspective. J Cell Biochem 2003;88:57-75

9 Lund RD, Ono SJ, Keegan DJ, et al. Retinal transplantation: progress and problems in clinical application. J Leukoc Biol 2003;74:151-60

10 Alteheld N, Roessler G, Vobig M, et al. The retina implant-new approach to a visual prosthesis. Biomed Tech (Berl) 2004;49:99-103 